\title{
Distribution of mitochondria in reconstructed mouse oocytes
}

\author{
Helena Fulka \\ Faculty of Natural Sciences, Charles University, Vinicna 7, 12844 Prague 2, Czech Republic and Institute of \\ Animal Production, POB 1, CS-104 01 Prague 10, Czech Republic
}

Correspondence should be addressed to H Fulka at the Institute of Animal Production; Email: hell.f@seznam.cz

\begin{abstract}
It has been suggested that nucleus replacement (transfer) may be used as an efficient oocyte therapy in order to prevent transmission of mutated mitochondrial DNA from mother to offspring in humans. The essential and not yet answered question is how mitochondria surrounding the karyoplast will be distributed in the newly reconstructed oocytes. In our model experiments, we have evaluated the distribution of mitochondria in reconstructed immature mouse oocytes when germinal vesicle karyoplasts, with labeled mitochondria, were fused to unlabeled cytoplasts. The penetration of mitochondria from karyoplasts into cytoplasts can be detected almost immediately after the beginning of fusion. In immature reconstructed oocytes, mitochondria are first located in the oocyte center but they are homogenously distributed within the whole cytoplasm before the completion of maturation. Fusion of oocytes at different stages of maturation suggests that the speed of mitochondria distribution is cell cycle dependent.

Reproduction (2004) 127 195-200
\end{abstract}

\section{Introduction}

At present, it is assumed that germinal vesicle (GV) transfer from developmentally compromised oocytes or from oocytes with mutated mitochondrial DNA (mtDNA) into enucleated donor immature oocytes may be an ethically acceptable procedure with a significant clinical impact (Report from the UK Department of Health: www.dch. gov.uk/cegc/stemcellreport.htm; Trounson, 2001). The technique of karyoplast exchange between mouse zygotes was developed 30 years ago (McGrath \& Solter 1983), and more recently similar approaches were tested for their potential in certain oocyte therapies in human assisted reproduction (Trounson 2001). Thus, it is suggested that the transfer of a GV-karyoplast from one oocyte into a cytoplast, obtained after the enucleation of a donor oocyte, may be applicable in the following cases: (i) if the patient's oocytes are morphologically abnormal; (ii) if the oocytes repeatedly fail to mature or develop after fertilization; (iii) to prevent transmission of mitochondria-related diseases and (iv) to prevent chromosomal abnormalities in oocytes obtained from older patients (Cummins 1998, Trounson 2001). The use of these techniques is new, especially in humans, but results from laboratory animals clearly show that GV transfer is technically feasible with a relatively high rate of maturation of the reconstructed oocytes (Takeuchi et al. 1999, 2001, Zhang et al. 1999,
Palermo et al. 2002). However, it is not known how these oocytes will develop when matured and subsequently fertilized. For successful enucleation, the surrounding cumulus cells must be removed. It is well known that in vitro cultured oocytes are developmentally compromised and this will certainly be enhanced when cumulus cells are absent (Moor et al. 1998, Russell 2001, Trounson et al. 2001). Up to now, mitochondria were exclusively transferred into oocytes by microinjection; thus it is not known how they will be distributed in a reconstructed cell after nucleus replacement (Van Blerkom et al. 1998, Barritt et al. 2001a). This would be an important issue if this approach were considered as a possible way to prevent the manifestation of mitochondria-related diseases. In our model experiments we have studied the distribution of mitochondria in oocytes reconstructed by induced fusion. The results showed that, at least in the mouse, the distribution of mitochondria is evidently uniform. Moreover, our results also show that the speed of mitochondria distribution in fused cells depends on the stage of the cell cycle.

\section{Materials and Methods}

Unless otherwise stated all chemicals were purchased from Sigma (Prague, Czech Republic). 


\section{Preparation of GV-karyoplasts and cytoplasts}

Immature oocytes were isolated from large antral follicles of 28-day-old ICR mice (Anlab, Prague, Czech Republic) stimulated $44 \mathrm{~h}$ previously with $5 \mathrm{IU}$ of equine chorionic gonadotropin. The animals were kept under standard conditions $\left(22^{\circ} \mathrm{C}, 60 \%\right.$ relative humidity, lights on from $0700 \mathrm{~h}$ to $1900 \mathrm{~h}$ ). All manipulations were performed at $37^{\circ} \mathrm{C}$ in air unless otherwise stated. To prevent GV breakdown (GVBD), oocytes were manipulated in M2 medium supplemented with dibutyryl cyclic AMP (dbcAMP) $(150 \mu \mathrm{g} / \mathrm{ml})$. Before enucleation, their zonae pellucidae were removed by pronase treatment $(0.5 \%$ in PBS, $10 \mathrm{~min}$ ) and oocytes were incubated in M2 containing cytochalasin D $(5 \mu \mathrm{g} / \mathrm{ml})$ and nocodazole $(3 \mu \mathrm{g} / \mathrm{ml})$ for $30 \mathrm{~min}$. The oocytes were then enucleated exactly as described previously by Karnikova et al. (1998). Briefly, oocytes were first elongated in a very narrow pipette so that the GV was located at one end. Next, that part of the oocyte with GV surrounded by a minimum amount of cytoplasm and enclosed with the oocyte plasma membrane was cut off with a glass needle. Then both parts, GV-karyoplast and the cytoplast, were cultured in M199 containing dbcAMP $(150 \mu \mathrm{g} / \mathrm{ml})$, gentamicin $(25 \mu \mathrm{g} / \mathrm{ml})$, Na-pyruvate $(0.2 \mathrm{mM})$ and BSA $(4 \mathrm{mg} / \mathrm{ml})$ in an atmosphere of $5 \% \mathrm{CO}_{2}$ in air at $37^{\circ} \mathrm{C}$ for $2-3 \mathrm{~h}$ before karyoplast labeling and fusion. Karyoplasts were incubated for $30 \mathrm{~min}$ at $37^{\circ} \mathrm{C}$ in $5 \% \mathrm{CO}_{2}$ in air in M199 supplemented with MitoTracker GreenFM (200 nM) (Molecular Probes, Eugene, OR, USA) to label the mitochondria. Then karyoplasts were washed several times in M2 and agglutinated to immature cytoplasts in PBS containing phytohemagglutinin $(\mathrm{PHA} ; 300 \mu \mathrm{g} / \mathrm{ml})$. The close contact between both parts was achieved by pipetting. Thereafter, the agglutinated components were transferred into a polyethylene glycol solution (PEG; $M_{\mathrm{r}} 1000 ; 1 \mathrm{~g} / \mathrm{ml}$ of M199) and incubated in it for 50-55 s (Fulka et al. 1995). After an extensive washing in M2 the agglutinated components were cultured in M199 with dbcAMP for $30 \mathrm{~min}$ and thereafter transferred into M199 without dbcAMP to permit GVBD and oocyte maturation. Fusion products were periodically inspected $(15,30,60 \mathrm{~min}, 3 \mathrm{~h}$ and $12 \mathrm{~h}$ ) under the fluorescence microscope (FITC filter excitation $490 \mathrm{~nm}$ ).

\section{Oocyte-to-oocyte fusion}

From our preliminary experiments it was evident that the speed of mitochondria distribution in reconstructed cells is cell cycle dependent. To study this in more detail the following zona-free oocyte $\times$ oocyte PEG-induced fusion combinations were devised: GV $\times$ GV (labeled) - cultured for up to $12 \mathrm{~h}$ in M199 with dbcAMP; GV (labeled) $\times$ metaphase I (MI) - fusion products were cultured for up to $12 \mathrm{~h}$ in M199 with dbcAMP; MI $\times$ MI (labeled) - cultured for up to $12 \mathrm{~h}$ in M199 without dbcAMP.

The oocytes were isolated from large antral follicles as described above. When immature oocytes were used, the maturation was prevented by dbcAMP. Maturing oocytes were obtained after $6 \mathrm{~h}$ culture in M199 without dbcAMP. About $30 \mathrm{~min}$ before the induction of fusion, mitochondria in chosen oocytes were labeled as described above. Thereafter, zonae pellucidae were removed from all oocytes by pronase treatment. The close contact of oocytes in a given combination was achieved in PHA solution and oocytes were then fused exactly as described above. Again, fused oocytes were observed periodically as described above. In each combination we have evaluated more than 50 fused cells.

\section{Results}

In total, we have bisected 350 immature oocytes, in 272 of which both components - karyoplast and cytoplast survived and were used for labeling and fusion (Fig. 1a). Fusion was successfully induced in 195 cases $(72 \%)$. The fusion between karyoplast and cytoplast was first detected $15 \mathrm{~min}$ post its induction (Fig. 1b). This was also confirmed by the penetration of labeled mitochondria from the karyoplast into the unlabeled cytoplast. Shortly thereafter, the GV enters into the cytoplast. After another $15 \mathrm{~min}$, fused cells have an oval shape with mitochondria preferentially located in the oocyte center. Then, the fusion products were removed from dbcAMP-supplemented medium and transferred into a normal medium to permit the process of maturation of these reconstructed oocytes. GVBD was generally completed within the next $2 \mathrm{~h}$ and this was accompanied by the gradual dispersion of mitochondria from the oocyte center into the whole cytoplasm (Fig. 1c). After another $10-11 \mathrm{~h}$ of culture, the reconstructed oocytes completed meiotic maturation and were arrested in metaphase II with the first polar body extruded $(150 / 195 ; 77 \%)$; there was remarkably uniform distribution of mitochondria in the cytoplasm (Fig. 1d). In conclusion, our results show that mitochondria surrounding GVs in karyoplast rapidly disperse in the immature cytoplasm producing a uniform distribution at the end of maturation.

In the next experiments we fused oocytes at different stages of maturation. In this case one whole oocyte was labeled with MitoTracker whilst the other one was not. When two GV-stage oocytes were fused to each other the penetration of mitochondria from labeled into unlabeled cytoplasm was very rapid (Fig. 2a) and after $1 \mathrm{~h}$ post induction of fusion, mitochondria were almost uniformly distributed in the giant cell cytoplasm (Fig. 2b). However, a completely different situation was observed when two metaphase oocytes were fused. The labeled and unlabeled cytoplasms were sharply separated and two distinct hemispheres were visible even after $3 \mathrm{~h}$ post induction of fusion (Fig. 2c). Judged according to the movement of GV, the immature cytoplasm always enters into maturing cytoplasm in fusion combination GV $\times M$ (Fulka et al. 1989). Therefore we have labeled an immature oocyte which 

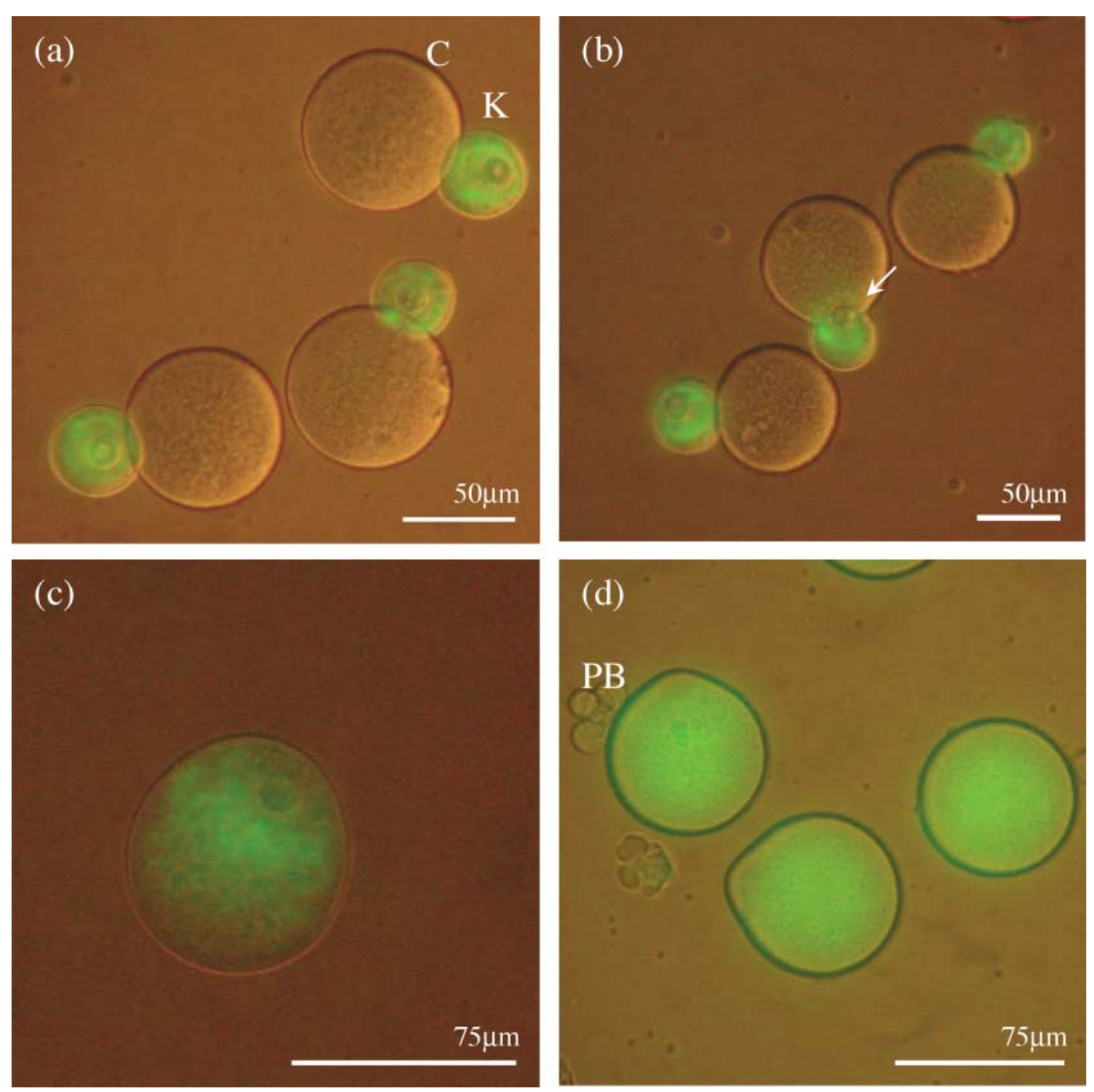

Figure 1 Distribution of mitochondria in mouse oocytes reconstructed by fusion of labeled germinal vesicle (GV)-karyoplast to an unlabeled cytoplast. (a) Cytoplasts (C) and GV-karyoplasts (K) before the induction of fusion. Interference contrast combined with fluorescence. (b) Labeled GV-karyoplast fused to an unlabeled cytoplast. The penetration of mitochondria (green color, arrow) can be detected within 15-30 min post induction of fusion. Interference contrast combined with fluorescence. (c) Labeled GV-karyoplast fused to an unlabeled cytoplast. The reconstructed oocyte was observed $60 \mathrm{~min}$ post induction of fusion. Note the localization of mitochondria in the oocyte center. Interference contrast combined with fluorescence. (d) When reconstructed oocytes reach the second metaphase stage the dispersion of mitochondria in the cytoplasm is uniform. PB, first polar body. Interference contrast combined with fluorescence.

was then fused to the metaphase oocyte. The movement of mitochondria from an immature into the maturing cytoplasm confirmed our previous observations. Here, mitochondria were quickly distributed in the giant cell cytoplasm with an almost uniform dispersion after $3 \mathrm{~h}$ (Fig. 2d). In all the above cases, mitochondria were uniformly dispersed in the giant cell cytoplasm after $12 \mathrm{~h}$ of culture. The addition of different cytoskeleton inhibitors (nocodazole, colcemid) did not significantly change the speed of mitochondria distribution (not shown).

In conclusion, our results clearly show that mitochondria transferred from the GV-karyoplast into the cytoplast are uniformly distributed in the reconstructed cell.
The speed of their distribution is cell cycle dependent with much faster distribution in interphase cells.

\section{Discussion}

The transfer of the cytoplasm from a donor oocyte into a recipient oocyte by injection has been successfully used in assisted human reproduction to rescue those oocytes that had repeatedly failed to develop after fertilization (Cohen et al. 1997, Barritt et al. 2001a). Observations of injected mitochondria indicate their gradual dispersion from the site of injection but these observations also show that they are not evenly distributed in the oocyte cytoplasm even after a relatively long culture 

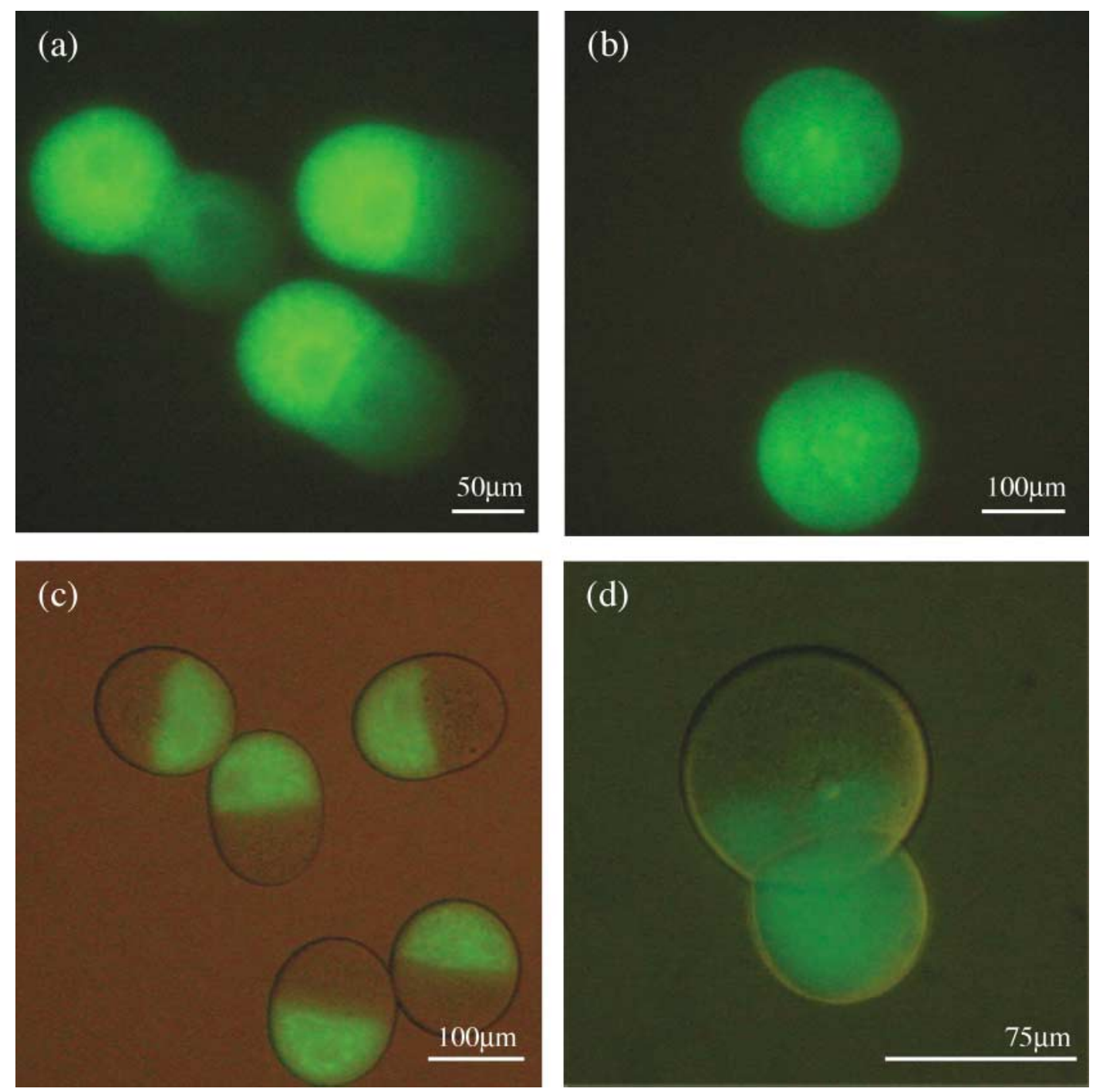

Figure 2 The rate of distribution of mitochondria in fused oocytes is dependent on the stage of the cell cycle. (a) Fusion of a labeled GV stage and an unlabeled oocyte. The penetration of mitochondria into unlabeled cytoplasm is very rapid (30 min). Fluorescence. (b) Fusion of a labeled GV stage and an unlabeled oocyte. Mitochondria are uniformly dispersed in the giant oocyte cytoplasm within $3 \mathrm{~h}$. Fluorescence. (c) Fusion of a labeled and an unlabeled metaphase oocyte. The labeled and unlabeled hemispheres are evident even after $3 \mathrm{~h}$ post fusion. Interference combined with fluorescence. (d) Labelled GV stage oocyte fused to an unlabeled metaphase oocyte. The mitochondria quickly penetrates into unlabeled cytoplasm $(30 \mathrm{~min})$. Interference contrast combined with fluorescence.

(Van Blerkom et al. 1998, Barritt et al. 2001b). Moreover, it has also been shown that mitochondria can be disproportionally distributed to blastomeres as embryos cleave (Van Blerkom et al. 2000, Barritt et al. 2001b). This would be potentially dangerous when nuclear replacement techniques were used, for example to eliminate mutated mitochondria - the defective cytoplasm should be completely replaced with a normal cytoplasm. However, the karyoplast will always be surrounded by at least some cytoplasm that will also contain mitochondria. If mitochondria surrounding the nucleus (GV) in the karyoplast remained located in a distinct area in the reconstructed oocyte, it is possible that the embryoblast would contain mostly mitochondria with mutated DNA and thus the disease would be manifested again.

In our mouse model experiments we show that transferred mitochondria are uniformly distributed in reconstructed cells. It is, however, possible that the situation in human oocytes will be different, but in our opinion this probability is rather low. Moreover, if used in humans we can expect that oocytes with zonae pellucidae will be manipulated (Palermo et al. 2002). We have, however, not found a difference in the pattern of mitochondria distribution when zona intact oocytes were used (not shown). Another problem could be the amount of mitochondria transferred. In immature oocytes the mitochondria are preferentially accumulated around GVs and in 
the cortical region (Calarco 1995, Van Blerkom et al. 1998). As it is accepted that for the manifestation of certain diseases the amount of mutated mtDNA can be as low as $10 \%$, this may represent a crucial problem (Wallace 1999). To avoid this, karyoplasts must contain a minimum amount of the surrounding cytoplasm. Another possibility is the use of specific mtDNA toxins, which will not damage nuclear DNA, or to expose karyoplasts to some oxidative agents such as $\mathrm{H}_{2} \mathrm{O}_{2}$. But in this case, there is a potential danger that damaged mitochondria will trigger apoptotic processes in the reconstructed cells (Liu et al. 2000a,b). Eventually, the centrifugation of oocytes may lead to the production of mitochondria-free GV-karyoplasts (Van Blerkom et al. 1998).

Our results also showed that the speed of mitochondria distribution is cell cycle dependent. Although this problem has a minimum of significance in our current experiments, it is potentially important in other nucleus replacement schemes, e.g. when the transfer of nuclei is carried out just before the cell cleaves. This may eventually result in two daughter cells, one of which will contain more transferred (and mutated) mitochondria than the other.

In our study, we concentrated on the distribution of mitochondria in reconstructed mammalian oocytes. However, with this approach, some other infertility problems might also be solved - GV could be transferred into normal cytoplasm when the original cytoplasm lacks some essential molecules e.g. proteins, RNAs (Levran et al. 2002). It is, however, well known that the development of in vitro matured oocytes is rather compromised after fertilization (Moor et al. 1998). We did not evaluate the development of our reconstructed embryos. This problem could be solved by a sequential nucleus transfer (Liu et al. 2003).

In conclusion, our results show that distribution of mitochondria in reconstructed mouse immature oocytes is rather rapid and uniform, but there are some other issues which are not yet fully solved. The most important would be the heteroplasmy resulting from a mixture of karyoplast and cytoplast mitochondria (St. John 2002). Secondly, it is not known if karyoplast mitochondria would have a replication advantage, and would therefore again dominate in all or some tissues of the children so born. This may be solved eventually when mitochondria-free karyoplasts are produced. But even in this case we must realize that the cytoplasm and the nucleus do not function as two independent units and that many nuclear genes are involved in mitochondrial biogenesis (Zeviani et al. 2003). Lastly, it has been well documented in laboratory animals that the source of cytoplasm may influence the activity of certain imprinted genes (Hawes et al. 2002). It is not known whether the same is true for humans. There is a need for additional experiments in laboratory and domestic mammals to confirm the safety of nucleus replacement procedures in human assisted reproduction.

\section{Acknowledgements}

HF is supported by grants from GACR 524/02/0032 and MSMT LN OOA 065.

\section{References}

Barritt JA, Willadsen S, Brenner C \& Cohen J 2001a Epigenetic and experimental modifications in early mammalian development. Part II. Cytoplasmic transfer in assisted reproduction. Human Reproduction Update 7 428-435.

Barrit JA, Brenner CA, Malter HE \& Cohen J 2001b Mitochondria in human offspring derived from ooplasmic transplantation. Human Reproduction 16 513-516.

Calarco P 1995 Polarization of mitochondria in the unfertilized mouse oocyte. Developmental Genetics 16 36-43.

Cohen J, Scott R, Schimmel T, Levron J \& Willadsen S 1997 Birth of infant after transfer of anucleate donor cytoplasm into recipients eggs. Lancet 350 186-187.

Cummins J 1998 Mitochondrial DNA in mammalian reproduction. Reviews in Reproduction 3 172-182.

Fulka J Jr, Flechon B \& Flechon JE 1989 Fusion of mammalian oocytes: SEM observations of surface changes. Reproduction Nutrition Development 29 551-558.

Fulka J Jr, Moor RM \& Fulka J 1995 Mouse oocyte maturation: meiotic checkpoints. Experimental Cell Research 219 414-419.

Hawes SM, Sapienza C \& Latham KE 2002 Ooplasmic donation in humans. The potential for epigenetic modifications. Human Reproduction 17 850-852.

Karnikova L, Urban F, Moor RM \& Fulka J Jr 1998 Mouse oocyte maturation: the effect of modified nucleocytoplasmic ratio. Reproduction Nutrition Development 38 665-670.

Levran D, Farhi J, Nahun H, Clezerman M \& Weissmann A 2002 Maturation arrest of human oocytes as a cause of infertility. Human Reproduction 17 1604-1609.

Liu L \& Keefe DL 2000a Cytoplasm mediates both development and oxidative-induced apoptotic cell death in mouse zygotes. Biology of Reproduction 62 1828-1834.

Liu L, Trimarchi JR \& Keefe DL $2000 b$ Involvement of mitochondria in oxidative stress-induced cell death in mouse zygotes. Biology of Reproduction 62 1745-1753.

Liu H, Chang HC, Zhang J, Grifo J \& Krey LC 2003 Metaphase II nuclei generated by germinal vesicle transfer in mouse oocytes support embryonic development to term. Human Reproduction 18 1903-1907.

McGrath J \& Solter D 1983 Nuclear transplantation in the mouse by microsurgery and cell fusion. Science 220 1300-1302.

Moor RM, Dai F, Lee C \& Fulka J Jr 1998 Oocyte maturation and embryonic failure. Human Reproduction Update $\mathbf{4}$ $223-236$.

Palermo GD, Takeuchi T \& Rosenwaks Z 2002 Technical approaches to correction of oocyte aneuploidy. Human Reproduction 17 2165-2173.

Russell JB 2001 In vitro oocyte maturation. In Assisted Fertilization and Nuclear Transfer in Mammals, pp 67-79. Eds DP Wolf \& $M$ Zelinsky-Wooten. Totowa, NJ: Humana Press.

St John JC 2002 Ooplasm donation in humans. The needs to investigate the transmission of mitochondrial DNA following cytoplasmic transfer. Human Reproduction 17 1954-1958.

Takeuchi T, Ergun B, Huang TH, Rosenwaks Z \& Palermo GD 1999 A reliable technique of nuclear transplantation of immature mouse oocytes. Human Reproduction 14 1312-1317.

Takeuchi T, Gong J, Veeck LL, Rosenwaks Z \& Palermo GD 2001 Preliminary findings in germinal vesicle transplantation of immature human oocytes. Human Reproduction 16 730-736.

Trounson A 2001 Nuclear transfer in human medicine and animal breeding. Reproduction Fertility and Development 13 31-39. 
Trounson A, Anderiesz C \& Jones G 2001 Maturation of human oocytes in vitro and their developmental competence. Reproduction $12151-75$.

Van Blerkom J, Sinclair J \& Davis P 1998 Mitochondrial transfer between oocytes: potential applications of mitochondrial donation and the issue of heteroplasmy. Human Reproduction 13 2857-2868.

Van Blerkom J, Davis P \& Alexander S 2000 Differential mitochondrial distribution in human pronuclear embryos leads to disproportionate inheritance between blastomeres: relationship to microtubular organization, ATP content and competence. Human Reproduction 15 2621-2633.

Wallace DC 1999 Mitochondrial diseases in man and mouse. Science 283 1482-1488.
Zeviani M, Spinazzola A \& Carelli V 2003 Nuclear genes in mitochondrial disorders. Current Opinion in Genetics and Development $13262-270$

Zhang J, Wang CW, Krey L, Liu H, Meng L, Blaszcyk A, Adler A \& Grifo J 1999 In vitro maturation of human preovulatory oocytes reconstructed by germinal vesicle transfer. Fertility and Sterility $\mathbf{7 1}$ $726-731$.

Received 25 July 2003

First decision 11 September 2003

Revised manuscript received 6 November 2003

Accepted 12 November 2003 